\title{
A Prospective Single-Arm Investigation Assessing Performance of a Multifunctional Foot Cream in Subjects with Tinea Pedis Interdigitalis and Heel Cracks, Calluses and/or Dry Feet
}

\author{
Martin Lundvall1, Kaj Stenlöf ${ }^{2}$, Jan Faergemann ${ }^{3}$ \\ ${ }^{1}$ Martin Lundvall, Specialist General Practitioner, Clinical Trials Unit, University Hospital of Örebro, Örebro, Sweden \\ ${ }^{2}$ Department of Medicine, Carlanderska Hospital, Göteborg, Sweden \\ ${ }^{3}$ Dermatology, Sahlgrenska Academy, University of Gothenburg, Göteborg, Sweden \\ Email: jan.faergemann@derm.gu.se
}

How to cite this paper: Lundvall, M., Stenlöf, K. and Faergemann, J. (2020) A Prospective Single-Arm Investigation Assessing Performance of a Multifunctional Foot Cream in Subjects with Tinea Pedis Interdigitalis and Heel Cracks, Calluses and/or Dry Feet. Journal of Cosmetics, Dermatological Sciences and Applications, 10, 20-27.

https://doi.org/10.4236/jcdsa.2020.101003

Received: February 15, 2020

Accepted: March 13, 2020

Published: March 16, 2020

Copyright $\odot 2020$ by author(s) and Scientific Research Publishing Inc. This work is licensed under the Creative Commons Attribution International License (CC BY 4.0).

http://creativecommons.org/licenses/by/4.0/

\section{(c) (i) Open Access}

\begin{abstract}
This prospective, open-label, single-arm investigation evaluated the performance of a multifunctional foot cream used twice daily for 8 weeks in the combined treatment of mild/moderate tinea pedis interdigitalis (TPI) and heel cracks, calluses and/or dry feet in 46 adult subjects. All subjects showed reductions in the severity of symptoms of both TPI (53.7\% reduction) and heel cracks, calluses and/or dry feet (51.6\% reduction), beginning already after 2 weeks of treatment, with reduction continuation and improvement after 4 and 8 weeks of treatment. Negative mycological cultures were obtained post-treatment in $85.7 \%$ of subjects, while $35.7 \%$ of subjects also obtained mycological cure (negative mycological culture + negative $\mathrm{KOH}$ test). Post-treatment questionnaires showed improved quality of life, good treatment tolerability, easy use, and that $69 \%$ of subjects would recommend the product. The results reveal that the foot cream is safe and effective in the combined treatment of TPI and heel cracks, calluses and/or dry feet.
\end{abstract}

\section{Keywords}

Tinea Pedis Interdigitalis, 1,5-Pentanediol, Heel Cracks, Calluses, Dry Feet

\section{Introduction}

Tinea pedis interdigitalis (TPI: athlete's foot) is a superficial inflammatory infec- 
tion of the skin on the foot caused by dermatophyte fungi [1] and is characterized by whiteish macerated skin, erythema (itchy or asymptomatic) and blister/cracks between the toes [2]. TPI is more common than the other clinical subtypes of moccasin and vesiculobullous tinea pedis [3]. Patients with TPI can also experience other foot problems, including heel cracks (rhagades), calluses (hyperkeratosis) and/or dry feet [4] [5].

Several TPI patients are refractory to treatment and those who respond to treatment frequently show recurrence [6]. Treatment is necessary to reduce symptoms, reduce the risk of secondary bacterial infections, and prevent spread to other body sites or individuals [1] [7]. The current prospective, single-arm investigation evaluated the clinical performance of a multifunctional foot cream used twice daily for 8 weeks in the combined treatment of subjects with mild to moderate TPI and heel cracks, calluses and/or dry feet.

\section{Materials and Methods}

The foot cream (Nåva Foot Cream, Salvequick/Salvelox Foot Rescue: Natumin Pharma AB, Sweden) is a multifunctional foot cream with 25\% 1,5-pentanediol (which has water binding and antimicrobial properties) [6] [8] [9] and Artemisia Abrotanum extract $(0.05 \%)$ for combination treatment of fungi (including those causing TPI) as well as heel cracks, calluses and/or dry feet.

After signing an Informed Consent Form, prospective adult subjects were evaluated at Visit 1 (Screening) for investigation eligibility regarding inclusion and exclusion criteria. During Visit 1, investigators performed a rating of symptoms using the Investigator's Global Assessment Scale for the eight clinical signs and symptoms of TPI (erythema, erosions, macerations, pruritus, fissures, burning/stinging, hyperkeratosis, odor) and the Investigator's Global Assessment Scale for heel cracks, calluses and dry feet. If the test scores reflected eligibility, a mycological culture sample was taken from the subject's (worst affected) foot. Subjects were enrolled if the culture was positive for dermatophytes: the timing of the return of these culture results determined the duration of the 3 to 5 week interval between Visit 1 (Screening) and Visit 2 (Baseline).

Treatment began at Visit 2 (Baseline) and continued for 8 weeks (Visit 5), with a treatment halt 2 days prior to Visit 5 to prevent contamination of cultures. Subjects applied one pump per foot of the foot cream twice daily (morning and evening) on dry and clean feet. During the investigation subjects were asked to note applications and other experiences in a Notebook. Subjects returned for follow-up visits in which the two clinical scores were assessed after 2 weeks (Visit 3), after 4 weeks (Visit 4) and after 8 weeks (Visit 5, end of investigation) of treatment. The Dermatology Life Quality Index (DLQI) (with 10 questions about the impact of skin disease and quality of life [10]) was administered at Baseline and at end of investigation. A photo of the foot was taken for documentation purposes at Visits 2 through 5.

Sample size calculation and statistics. This study is associated with a statistical power of $90 \%$ (two-sided tests). The sample size calculation of the primary 
endpoint is based on the assumption of a reduction in the Total Mean Score (TMS) from 8.8 to 5.9 (with a standard deviation of 6.1 and 5.2 respectively). This mean reduction corresponds to $33 \%$. The calculation indicates that a sample size of 43 individuals is required. The sample size estimation of the secondary endpoint is based on a reduction in the Dermatology Life Quality Index Scale from 9.9 to 4.9 (with a standard deviation of 5 in both cases). Subsequently, a $50 \%$ reduction is expected in the Dermatology Life Quality Index Scale between baseline and the final week.

In order to detect the mean reduction as formulated as the primary endpoint, this investigation will include 43 subjects plus 5 individuals to take a $10 \%$ drop-out rate into account. Thus, a total of 48 subjects will be included in this investigation.

Formal statistical analysis was performed for the primary efficacy endpoint, reduction in total mean score of clinical signs and symptoms of TPI $\geq 33 \%$. All other tests were descriptive. T-tests were used for variables with normal distribution and non-parametric Wilcoxon-signed rank test when this was not the case.

The investigation received a positive decision after review from an Ethical Committee prior to investigation start, and was performed in accordance with the MDD93/42/EEC Annex X, ISO 14155 and the Declaration of Helsinki.

\section{Results}

\subsection{Subjects}

A total of 46 subjects were enrolled from 3 investigational sites, with 42 in the full analysis set (FAS) and 40 in the per protocol analysis set (PPS). In Figure 1 you will see a patient with typical tinea pedis interdigitalis.

In the FAS, 33 subjects (78.6\%) were male and the mean age was $56 \pm 12$ years. A third of the subjects had a history with previous fungal infection. Three subjects terminated investigation participation early (2 lost to follow-up at Visit 4 and 1 withdrawing consent at Visit 3).

\subsection{Primary endpoint}

As differences between FAS and PPS were minimal, only FAS results are summarised. Table 1 shows that the 8-week treatment with the foot cream resulted in a significant difference in total mean score (TMS) of TPI clinical signs and symptoms of $4.8 \pm 2.2$ between Baseline and end of investigation; this total TMS reduction between Baseline and end of investigation was 53.7\%, thus exceeding the primary endpoint of a TMS reduction of $\geq 33 \%$. Table 2 shows mean and standard deviations (as well as minimum and maximum scores) obtained at each visit using the Global Assessment Scale for the eight clinical signs and symptoms of TPI. The treatment effect of the foot cream on TPI was already observable after 2 weeks of treatment, and this treatment effect continued and improved after 4 weeks of treatment and again after 8 weeks of treatment as shown in Figure 2. 


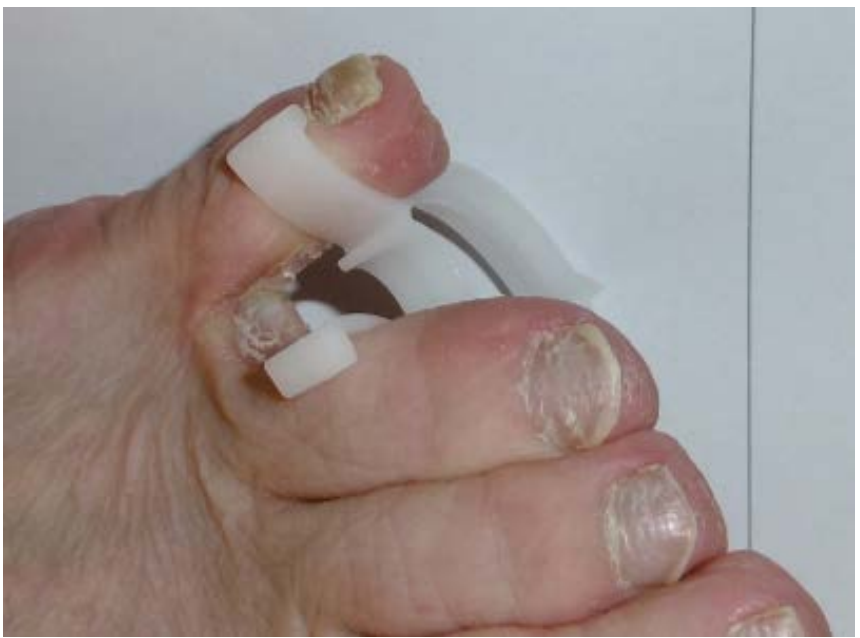

Figure 1. A study patient with typical tinea pedis interdigitalis.

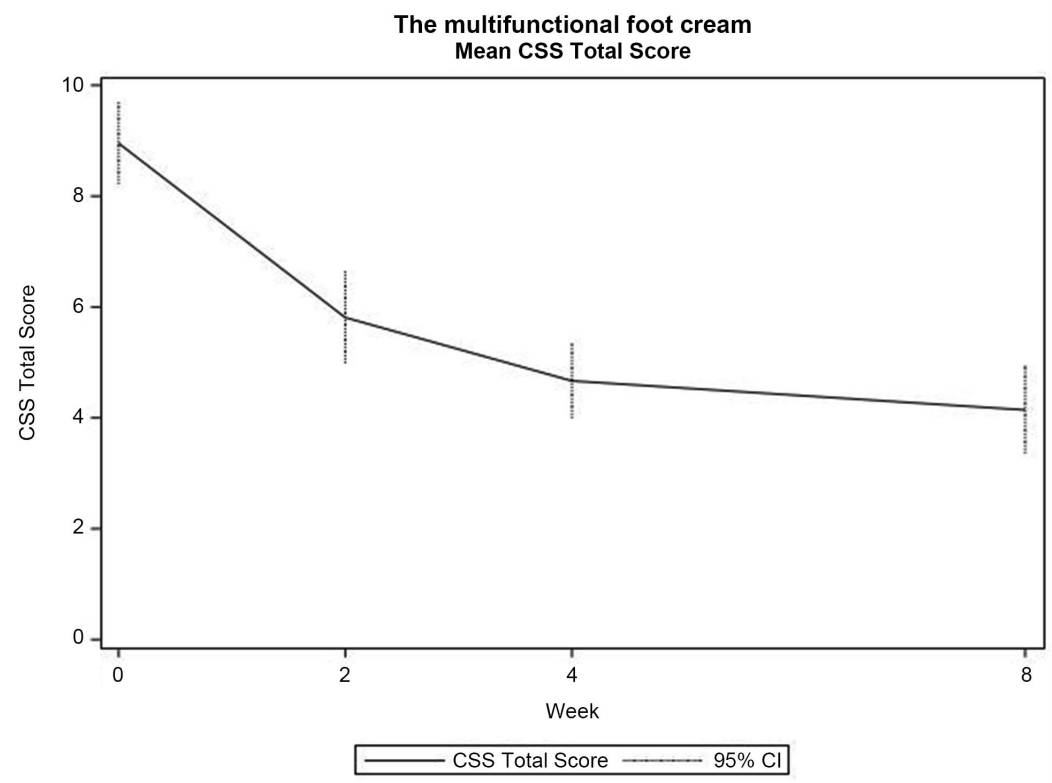

Figure 2. Decrease in total mean score for clinical signs and symptoms (CSS) of TPI.

Table 1. Total mean score. Reduction in total mean score for clinical signs and symptoms of tinea pedis interdigitalis from baseline to visit 5 .

\begin{tabular}{cccccc}
\hline N & Mean & SD & Minimum & Maximum & p-value \\
\hline 42 & 4.8 & 2.2 & 0 & 9 & $<0.001$ \\
\hline
\end{tabular}

Table 2. Total score of clinical signs and symptoms for tinea pedis interdigitalis by visit.

\begin{tabular}{cccccc}
\hline Visit & N & Mean & SD & Minimum & Maximum \\
\hline 2 & 42 & 9.0 & 2.3 & 6 & 14 \\
3 & 42 & 5.8 & 2.6 & 0 & 12 \\
4 & 42 & 4.7 & 2.2 & 0 & 8 \\
5 & 42 & 4.1 & 2.5 & 0 & 10 \\
\hline
\end{tabular}




\subsection{Secondary Endpoints}

The treatment with the multifunctional foot cream resulted in a difference in total scores on the Global Assessments Scale for severity of heel cracks, calluses, and dry feet between Baseline (Visit 2; $6.2 \pm 2.1$ ) and end of investigation (Visit $5 ; 3.0 \pm 1.8$ ); the total reduction between Baseline and end of investigation was $51.6 \%$. Figure 3 shows that the treatment effect of the foot cream upon heel cracks, calluses and dry feet was (as was the case with the treatment effect for TPI) already observable at 2 weeks of treatment (Visit 3), and that this treatment effect continued throughout the complete investigation. Each of the three conditions showed improvement; there was a $73.5 \%$ reduction in heel cracks, a $45.7 \%$ reduction in calluses, and a $43.2 \%$ reduction in dry feet between Baseline (Visit 2) and end of investigation (Visit 5).

Samples taken at the end of the investigation showed that $85.7 \%$ of subjects had a negative mycological culture and that $35.7 \%$ of subjects also had a negative $\mathrm{KOH}$ test (thus demonstrating a mycological cure) after 8 weeks of treatment. Treatment resulted in a significant (71.9\%) reduction of the DLQI score, from $5.4 \pm 3.3$ at Baseline to $1.5 \pm 1.0$ at end of investigation. The use of the foot cream was generally well accepted. However, treatment was judged as sticky by $50.0 \%$ of subjects and as very sticky by $23.8 \%$ of subjects. The majority of subjects (95.2\%) rated the overall treatment as well tolerable. One hundred percent of subjects assessed the device as easy to use, and $69 \%$ of the subjects would recommend this multifunctional foot cream to a friend for the treatment of TPI.

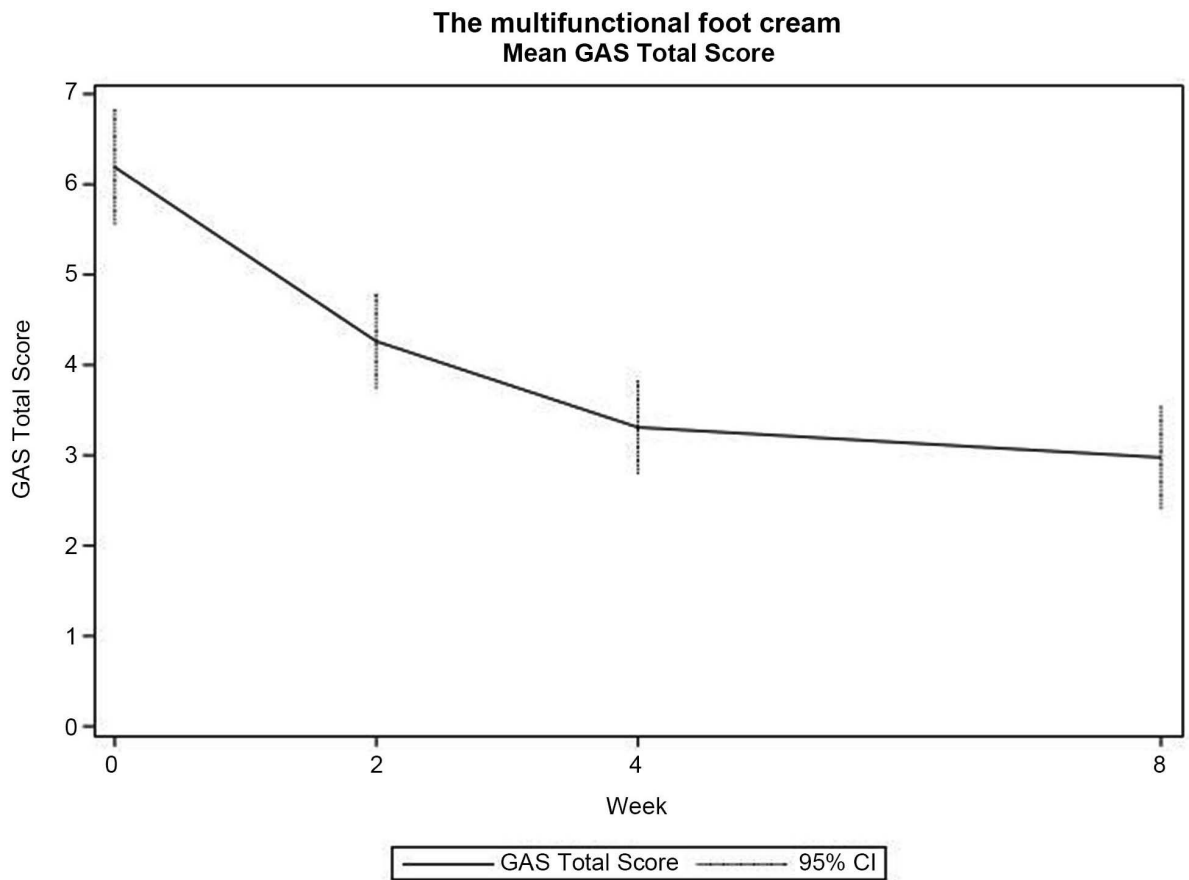

Figure 3. Decrease in mean global assessment scale score for heel cracks, calluses and dry feet. 


\subsection{Safety}

There were 9 subjects (20.0\%) who experienced at least 1 adverse event. There were a total of 19 adverse events (AEs); 9 of which were unique and 5 of these AEs were evaluated as device related (adverse device events): 4 cases of "Light burning feeling at feet" (in a single subject on 4 occasions, and which disappeared after 2 days use; probably related to the device) and 1 case of "Cold feet" (possibly related to the device). There were no serious AEs, no AEs leading to premature discontinuation of use of the device, and no device deficiencies reported during the investigation.

\section{Discussion}

TPI (Athlete's foot) is a common condition that is associated with discomfort and unpleasant appearance of the feet which often influences the quality of life for those affected [2]. The results of the DLQI reported in the current investigation show that even mild to moderate symptoms of the investigated skin conditions can have a profound impact on the quality of life of those suffering from these conditions as it influences choice of clothes, ability to do sports and other activities and may impact the social life and interactions with others. It is therefore encouraging to see that treatment with this multifunctional foot cream was not only effective on the TPI clinical signs and symptoms but also resulted in a statistically significant improvement of the DLQI, as the score was reduced by $71.9 \%$. The clinical signs and symptoms of TPI were reduced by $53.7 \%$ after 8 weeks' treatment. In addition, the treatment resulted in significant reductions of the severity of heel cracks, calluses and dry feet. Standard topical antifungal therapies, azoles and allylamines, are highly effective in the treatment of TPI, however, they have no or minor effects on heel cracks, calluses and dry feet [7]. This foot cream therefore offers a valuable treatment option for those suffering from mild to moderate TPI in combination with other foot problems such as heel cracks, calluses and dry feet

Mycological culture and $\mathrm{KOH}$-test was negative in $86 \%$ and $36 \%$ of subjects at end of investigation. However, even after a completed treatment, traces of the dermatophytes might still be remaining in samples and seen in microscopy. When assessing the relevance of these traces seen in the $\mathrm{KOH}$-test it should take into account that it might be dead fungi still remaining on/in the skin, thus giving a positive results even if the fungi is eliminated.

The clinical investigation showed that a treatment period of eight weeks is clinically relevant and that initial results are usually visible within two weeks, both on TPI and heel cracks, calluses and dry feet, with increasing effect after four and eight weeks of treatment.

Compliance with treatment was high during the investigation, as self-reported in the notebooks (only two of the 42 subjects used the device less than 11 times a week). The usability results show that subjects had a high satisfaction and acceptability of the product (69\% would recommend the product to a friend). The 
product was considered a bit sticky by a majority of subjects, but was still accepted, and overall the investigation subjects were in favour of the treatment.

The treatment has been demonstrated to be safe in the simultaneous combined treatment of TPI and heel cracks, calluses and dry feet. Observed AEs were few and mild, there were no serious AEs, no AEs leading to premature discontinuation of the device, and no device deficiencies reported during the study.

\section{Conclusion}

The results of this clinical investigation confirm that this multifunctional foot cream has a clinically significant effect when used as a combined treatment for TPI and for heel cracks, calluses and dry feet. The simultaneous combined therapeutic effect was seen both in the reduction of clinical signs and symptoms of TPI, and the reduction of severity of heel cracks, calluses and dry feet, and in improvements of the subject's quality of life. It is thereby confirmed that this foot cream is a multifunctional cream that provides a valuable treatment option for people suffering from mild to moderate TPI and heel cracks, calluses and dry feet. The safety and usability of the device are also confirmed, making it a useful and safe treatment for self-care in the home environment.

\section{Conflicts of Interest}

The authors declare no conflicts of interest regarding the publication of this paper.

\section{References}

[1] Leyden, J.J. and Kligman, A.M. (1978) The Interaction of Dermatophytes and Resident Bacteria. Archives of Dermatology, 114, 1466-1472. https://doi.org/10.1001/archderm.1978.01640220015004

[2] Crawford, F. and Hollis, S. (2007) Topical Treatments for Fungal Infections of the Skin and Nails of the Foot. Cochrane Database of Systematic Reviews. https://doi.org/10.1002/14651858.CD001434.pub2

[3] Karami, N., Alsterholm, M. and Faergemann, J. (2009) In Vitro Activity of Chlorhexidine and Pentane-1,5-Diol and Their Combination on Candida Albicans, Staphylococcus aureus and Propionibacterium acnes. Acta Dermato-Venereologica, 89, 514-515. https://doi.org/10.2340/00015555-0706

[4] Robbins, J.M. (2000) Recognizing, Treating, and Preventing Common Foot Problems. Cleveland Clinic Journal of Medicine, 67, 45-7+51-2+55-6. https://doi.org/10.3949/ccjm.67.1.45

[5] Kircik, L.H. and Onumah, N. (2014) Use of Naftifine Hydrochloride 2\% Cream and 39\% Urea Cream in the Treatment of Tinea Pedis Complicated by Hyperkeratosis. Journal of Drugs in Dermatology, 13, 162-165. https://www.ncbi.nlm.nih.gov/pubmed/24509966

[6] Faergemann, J. and Fredriksson, T. (1980) The Antimycotic Activity in Vitro of Five Diols. Sabouraudia, 18, 287-293. https://doi.org/10.1080/00362178085380491

[7] Becker, B.A. and Childress, M.A. (2018) Common Foot Problems: Over-the-Counter Treatments and Home Care. American Family Physician, 98, 298-303. 
https://www.ncbi.nlm.nih.gov/pubmed/30216025

[8] Sundberg, J.J. and Faergemann, J. (2008) A Comparison of Pentane-1,5-Diol to Other Diols for Use in Dermatology. Expert Opinion on Investigational Drugs, 17, 601-610. https://doi.org/10.1517/13543784.17.4.601

[9] Faergemann, J., Hedner, T. and Larsson, P. (2005) The in Vitro Activity of Pentane-1,5-Diol against Aerobic Bacteria. A New Antimicrobial Agent for Topical Usage? Acta Dermato- Venereologica, 85, 203-205.

https://doi.org/10.1080/00015550410026128

[10] Basra, M.K., Fenech, R., Gatt, R.M., Salek, M.S. and Finlay, A.Y. (2008) The Dermatology Life Quality Index 1994-2007: A Comprehensive Review of Validation Data and Clinical Results. The British Journal of Dermatology, 159, 997-1035.

https://doi.org/10.1111/j.1365-2133.2008.08832.x 